\title{
Adaptação cultural e linguística e validaçáo da Escala de Quedas de Morse
}

\author{
Cultural and linguistic adaptation and validation of the Morse Fall Scale \\ Adaptación cultural y lingüística y validación de la Escala de Caídas de Morse \\ Maria José Martins da Costa-Dias*; Pedro Lopes Ferreira**; \\ Alexandre Santos Oliveira****
}

\section{Resumo}

Enquadramento: A queda do doente é o incidente de segurança mais reportado no contexto hospitalar. As quedas têm óbvias consequências no doente, na família e nos profissionais de saúde. A prescrição de intervenções de prevenção deve basear-se na avaliação do risco de queda apresentado por cada doente, o qual pode ser avaliado através de escalas como a Escala de Quedas de Morse (MFS). Esta escala encontra-se introduzida em diversos hospitais, no entanto sem qualquer validação formal até agora.

Objetivos: Avaliar o grau de reprodutibilidade da MFS em contexto hospitalar e analisar a sua validade através das correlações com outros instrumentos de medida.

Metodologia: O estudo decorreu em dois hospitais da zona de Lisboa e participaram 120 enfermeiros que aplicaram a MFS a 200 doentes. A cada doente foram realizadas três avaliações da MFS, por três enfermeiros diferentes, obtendo-se 600 avaliações.

Resultados: A versão portuguesa obtida é semântica e culturalmente equivalente à original, com uma boa fiabilidade (coeficiente de correlação intraclasse de 0,838 e concordância entre observadores, avaliada pela média dos coeficientes K, entre 0,615 e 0,964) e validade convergente satisfatória.

Conclusão: Conclui-se recomendando a sua utilização nas organizações de prestação de cuidados hospitalares.

Palavras-chave: acidentes por quedas; controle de risco; serviços hospitalares.

\begin{abstract}
Background: Patients' falls are the most commonly reported safety incident in hospitals. Falls have clear consequences on the patient, family and health professionals. Prevention interventions should be prescribed based on each patient's fall risk assessment. This assessment may be performed using scales such as the Morse Fall Scale (MFS). Despite being used in several hospitals, this scale was never formally validated until now.

Objectives: To assess the degree of reproducibility of the MFS in hospital settings and examine its validity through correlations with other measuring instruments.

Methodology: The study was conducted in two hospitals in the Lisbon area using a sample composed of 120 nurses, who applied the MFS to 200 patients. Each patient was assessed three times with the MFS by three different nurses. A total of 600 assessments were performed. Results: The Portuguese version is semantically and culturally equivalent to the original, with good reliability (intraclass correlation coefficient of 0.838 and interobserver agreement between 0.615 and 0.964 , assessed using the mean kappa coefficient) and satisfactory convergent validity.

Conclusion: The Portuguese version is recommended for use in hospital context.
\end{abstract}

Keywords: accidental falls; risk management; hospital services.

*Aluna do Doutoramento em Enfermagem do Instituto de Ciências da Saúde da Universidade Católica Portuguesa. Enfermeira, Especialista em Saúde Infantil e Pediátrica,Hospital da Luz, 1500-650, Lisboa, Portugal [cdias@hospitaldaluz.pt]. Morada para correspondência: Rua Basílio Teles n ${ }^{\circ} .11,2^{\circ}$ Esq. 2720-063, Amadora, Portugal.

** Professor Associado com Agregaçãa, Diretor do Centro de Estudos e Investigação em Saúde da Universidade de Coimbra, Faculdade de Economia da Universidade de Coimbra, 165, 3004-512, Coimbra, Portugal [pedro@fe.uc.pt].

*** Aluno do Doutoramento em Enfermagem do Instituto de Ciências da Saúde da Universidade Católica Portuguesa. Enfermeiro, Especialista em Enfermagem MédicoCirúrgica, Hospital Curry Cabral, 1069-166, Lisboa, Portugal [enf.oliveira@gmail.com].

\section{Resumen}

Marco contextual: La caída de los pacientes es el incidente de seguridad más comunicado en el contexto hospitalario. Las caídas tienen consecuencias evidentes en los pacientes, la familia y los profesionales de la salud. La prescripción de las intervenciones para prevenir caídas debe basarse en la evaluación del riesgo de caída que presenta cada paciente, que puede ser evaluado mediante escalas como la Escala de Caídas de Morse (MFS). Esta escala se encuentra introducida en varios hospitales, sin embargo todavía no cuenta con una validación formal.

Objetivos: Evaluar el grado de reproducibilidad de la MFS en el contexto hospitalario y examinar su validez a través de las correlaciones con otros instrumentos de medición.

Metodología: El estudio se llevó a cabo en dos hospitales de la zona de Lisboa y participaron 120 enfermeros, que aplicaron la MFS a 200 pacientes. A cada paciente, tres enfermeros diferentes le realizaron tres evaluaciones de la MFS, de forma que se obtuvieron 600 evaluaciones.

Resultados: La versión portuguesa es semántica y culturalmente equivalente a la original y que tiene una buena fiabilidad (coeficiente de correlación intraclase de 0,838 y acuerdo entre observadores, evaluada mediante la media del coeficiente K, entre 0,615 y 0,964 ) y una validez convergente satisfactoria. De ello resulta que la versión portuguesa es semánticamente equivalente a la versión original. Conclusión: En conclusión, su uso se recomienda en las organizaciones de atención hospitalaria.

Palabras clave: accidentes por caídas; control de riesgo; servicios hospitalarios.

Recebido para publicação em: 23.03.13

Aceite para publicação em: 05.02 .14 


\section{Introdução}

A prevalência de quedas dos doentes é um indicador de segurança do doente a nível hospitalar e um indicador sensível aos cuidados de Enfermagem. A evidência da investigação e as estatísticas hospitalares sugerem que os doentes hospitalizados têm um risco maior de cair do que as pessoas na comunidade (Healey \& Scobie, 2007). A queda do doente é o incidente mais comum de segurança reportado a nível hospitalar (Healey \& Scobie, 2007; Oliver et al., 2007; Perell, Nelson, Goldman, Prieto-Lewis, \& Rubenstein, 2001), as quais têm custos a nível dos doentes, dos familiares e até dos próprios profissionais. Em termos humanos, podem provocar no doente angústia, dor, danos físicos, perda de confiança e perda de independência. A nível dos familiares, podem gerar reclamações formais e processos jurídicos de litígio com o hospital, sendo natural que fiquem insatisfeitos quando um familiar internado sofre uma queda que cause danos, pois esperam que o hospital seja um lugar seguro. A nível dos profissionais, apesar de se esforçarem para que os doentes mais vulneráveis não caiam, constitui uma constante fonte de ansiedade e desgaste sempre que um doente sofre lesões provocadas por uma queda (Healey \& Scobie, 2007; Kohn, Corrigan, \& Donaldson, 2000; Oliver, Daly, Martin, \& McMurdo, 2004; Perell et al., 2001).

A avaliação do risco de queda efetua-se através de escalas de avaliação de risco, ferramentas que atribuem valores numéricos a determinados fatores de risco (Healey \& Scobie, 2007), geralmente, somados de forma a predizerem se o doente tem um risco baixo, médio ou elevado de cair (Morse, 2009). Num relatório elaborado pela Inspeção Geral das Atividades em Saúde sobre as quedas em hospitais (Soares \& Almeida 2008) é referido que a Escala de Quedas de Morse (do inglês, Morse Fall Scale - MFS) é a escala mais utilizada nos hospitais portugueses, embora seja de realçar a sua utilização sem ter sido validada para a população portuguesa.

São objetivos deste estudo avaliar o grau de reprodutibilidade da MFS em contexto hospitalar e contribuir para a sua validade analisando as suas correlações com outros instrumentos de medida.

\section{Enquadramento}

As escalas de avaliação de risco têm sido assunto de revisão sistemática na literatura quanto à previsão do risco
(Morse, 2009; Oliver, Britton, Seed, Martin, \& Hopper, 1997). No entanto, apenas duas escalas foram testadas em diferentes grupos de doentes fora dos estudos de investigação originais (Oliver et al., 2004), sendo uma delas a MFS. Há, no entanto, que chamar a atenção para o facto de, mesmo as melhores escalas, poderem sub- ou hiper-predizerem o risco de queda (Oliver, 2008). Além disto, as escalas estão desenhadas para predizerem o risco, o que não é o mesmo que prevenir eficazmente as quedas. O score avaliado pelas escalas, por si só, não define intervenções. O objetivo das escalas de avaliação do risco de queda é triar de forma rápida as pessoas com risco de forma a se implementarem estratégias que previnam danos ao doente (Morse, 2006).

A MFS foi desenvolvida por Janice Morse em 1985, na Universidade de Alberta, no Canadá, com base num estudo de 100 doentes com quedas e 100 doentes sem queda selecionados de forma aleatória (Morse, 2009). Foi testada por outras organizações e autores de forma independente e destina-se ao adulto em geral (Morse, 2006). Esta escala consiste em seis itens que refletem os fatores de risco de queda (Morse, 2009; Morse, Morse, \& Tylko, 1989), tendo sido demonstrada a sua validade preditiva, bem como a sua fiabilidade entre avaliadores (Morse, 2006).

A MFS é particularmente importante no momento da admissão dos doentes, sobretudo nas pessoas com 65 anos ou mais anos de idade, nos doentes confusos ou com agitação psicomotora, nos doentes no pósoperatório, em doentes medicados com fármacos associados ao risco de queda, como psicofármacos, analgésicos estupefacientes ou anti-hipertensores (Leipzig,Cumming, \& Tinetti,1999a; Leipzig,Cumming, \& Tinetti, 1999b) entre outros, contribuindo para um adequado plano de intervenção a nível da prevenção das quedas dos doentes em ambiente hospitalar.

Segundo a autora da escala, a grande maioria dos enfermeiros (82,9\%) descreve esta escala como "rápida e fácil de usar" e estima que são precisos menos de 3 minutos para avaliar um doente (Morse, 2009; Morse et al.,1987).

Os doentes podem ter características diferentes em países e hospitais diferentes. Por isso, a explicação fornecida para cada item sobre a forma como deve ser entendida e interpretada a escala é fundamental para que todos possam utilizar a escala do mesmo modo. A revisão da literatura alerta para o facto de uma escala, mesmo testada num determinado hospital, poder não predizer adequadamente o risco de queda num hospital 
diferente (Morse, 2009). Portanto, com a disponibilização da versão portuguesa da MFS devidamente validada, poderemos avaliar o risco de queda de uma forma mais segura e ajustada à realidade portuguesa. A utilização da MFS, pelos enfermeiros portugueses, só será cientificamente válida quando for demonstrada a sua adaptação cultural e linguística e revelar uma boa reprodutibilidade entre avaliadores, assim como quando apresentar indicadores reconhecidos de validade.

\section{Metodologia}

A adaptação cultural e linguística para português da versão em língua inglesa da MFS foi iniciada após se ter obtido a autorização formal da autora. O processo seguido respeitou as normas da International Society for Pharmacoeconomics and Outcomes Research (ISPOR) Task Force for Translation and Cultural Adaptation (Wild et al., 2005), incluindo: (1) duas traduções independentemente realizadas por dois tradutores portugueses; (2) uma primeira versão de consenso; (3) uma retroversão realizada por uma tradutora de língua-mãe inglesa; (4) análise comparativa entre a retroversão e a versão original; (5) alterações resultantes desta análise comparativa; (6) revisão da primeira versão em português.

Esta primeira versão em português foi então submetida a uma revisão por um especialista, tendo- lhe sido solicitado que prestasse particular atenção aos termos técnicos incluídos no questionário. Para cada item da escala, foi-lhe apresentada a versão original assim como a tradução a que se tinha chegado, e foilhe pedido que nos informasse se se sentia satisfeito com a forma como este item estava traduzido ou, se pelo contrário, sugeria qualquer alteração. O referido especialista concordou com a totalidade da tradução apresentada. Uma vez que esta escala está desenhada para ser respondida por profissionais de Enfermagem, prescindiu-se de uma avaliação cognitiva e do teste de interpretação por parte de uma amostra de doentes. Por fim, a versão portuguesa da MFS foi submetida a um outro perito que, lendo-a, não propôs qualquer alteração do ponto de vista da língua e gramática portuguesa. Os itens da versão portuguesa da Escala de Morse podem ser lidos na Tabela 1.

A pontuação total da escala varia entre 0 e 125 pontos e os indivíduos são descriminados em função do risco de queda em: sem risco (0-24), baixo risco (25-50) ou alto risco $(\geq 51)$.

A fiabilidade foi testada em cada serviço em que decorreu o estudo, por grupos de três enfermeiros observadores, num total de 120, que avaliaram de forma independente o risco de queda no mesmo doente. Cada enfermeiro efetuou cinco avaliações. Foi dada uma explicação da escala de cerca de 15 minutos aos enfermeiros participantes por já estarem todos familiarizados com a utilização da escala.

Tabela 1

Itens da versão portuguesa da Escala de Quedas de Morse

\begin{tabular}{lc}
\hline Item & Pontuação \\
\hline 1. Historial de quedas; neste internamento urgência/ ou nos últimos três meses & 0 \\
Não & 25 \\
Sim & 0 \\
2. Diagnóstico(s) secundário(s) & 15 \\
Não & 0 \\
Sim & 15 \\
3. Ajuda para caminhar & 30 \\
Nenhuma/ajuda de enfermeiro/acamado/cadeira de rodas & 0 \\
Muletas/canadianas/bengala/andarilho & 20 \\
Apoia-se no mobiliário para andar & \\
4. Terapia intravenosa & 0 \\
Não & 10 \\
Sim & 20 \\
5. Postura no andar e na transferência & \\
Normal/acamado/imóvel & 0 \\
Debilitado & 15 \\
Dependente de ajuda & \\
6. Estado mental & \\
Consciente das suas capacidades & \\
Esquece-se das suas limitações & \\
\hline
\end{tabular}


A validade facial (de conteúdo) foi conferida por meio da realização de um teste de compreensão a um painel de peritos constituídos por seis profissionais de saúde. Procedeu-se a um teste cognitivo com o objetivo de avaliar a clareza, a compreensão, a relevância cultural, a inclusão de todos os conceitos bem como a redundância e ambiguidade dos itens utilizados. Os restantes testes da validação do estudo decorreram durante o primeiro trimestre de 2011, num hospital privado da grande Lisboa, identificado como A (dois serviços de internamento médicocirúrgico, um serviço de internamento de cuidados continuados e paliativos e uma unidade de cuidados intensivos) e num centro hospitalar igualmente de grande Lisboa, identificado como B (oito serviços de medicina, um serviço de pneumologia, um serviço de neurologia, três serviços de neurocirurgia, uma unidade cérebro vascular, um serviço de cirurgia cardiotorácica, um serviço de cirurgia vascular, um serviço de cardiologia e um serviço de cirurgia geral). A amostra foi constituída por 200 doentes e foi obtida através de um método não-probabilístico acidental, constituído pelos doentes que estavam acessíveis no momento do estudo, dos quais 110 (55\%) são do hospital A e 90 (45\%) do centro hospitalar B. Foram considerados critérios de inclusão uma idade do doente igual ou superior a 18 anos e o facto de os serviços já terem introduzido no padrão de registos a MFS, para garantir uma experiência de utilização da escala mais homogénea.

Na colheita de dados utilizou-se uma grelha de registo, em que se obtiveram a avaliação do risco de queda e a caracterização do doente observado, sendo mantido sempre o anonimato e a confidencialidade do doente. Seguiram-se os procedimentos éticos recomendados em ambos os locais do estudo e obtiveram-se as devidas autorizações. No tratamento estatístico dos dados, foi utilizado o programa estatístico SPSS ${ }^{\circledR}$ Statistical Package for the Social Siences, versão 18 para o Windows ${ }^{\circledR}$. Os resultados foram analisados através de medidas de correlação, de tendência central, de dispersão e de testes paramétricos.

A fiabilidade foi avaliada quanto à concordância dos resultados registados pelos enfermeiros (Terwee et al., 2007). Foi utilizado o coeficiente $\mathrm{K}$ de Cohen, sendo considerada uma concordância moderada o valor entre 0,41 e 0,60, uma concordância substancial o valor entre 0,61 e 0,80, e uma concordância quase perfeita o valor entre 0,81 e 1,00 (Kramer \& Feinstein, 1981).
A validade foi testada através da validade de critério e da validade de constructo. Na validade de critério, representando, na opinião do painel COSMIN (Mokkink et al.,2010), o grau segundo o qual os valores de um instrumento de medição são um reflexo adequado de um padrão-ouro, foi calculada a correlação entre a MFS e outros instrumentos de medida utilizados nos hospitais onde decorreu o estudo, isto é, a Escala de Coma de Glasgow e o Índice de Barthel. No entanto, não sendo possível neste caso, como em muitos outros, obter uma medida padrão que se sobrepusesse à MFS, fomos comparar alguns dos itens que constituem esta medida com os valores finais ou outros itens de outras medidas de referência. É o caso, por exemplo, da comparação do item da MFS referente ao estado mental que comparámos com a Escala de Glasgow e dos itens da MFS referentes ao andar e ao caminhar que relacionámos com alguns itens da Escala de Barthel.

A validade de construto, que ainda de acordo com o painel COSMIN (Mokkink et al., 2010) constitui o grau segundo o qual os valores de um instrumento de medição são coerentes com hipóteses previamente definidas ou com valores de grupos, foi testada pelo método dos grupos de contraste, ou seja, pela comparação entre valores de grupos diferentes, como a idade, o sexo, o historial de quedas, a agitação psicomotora e o tipo de medicação que o doente efetua. Tanto na validade de critério como na validade de construto, recorreu-se ao coeficiente de correlação de Spearman. Os seus valores variam de +1 (correlação positiva/direta perfeita), atravessando o 0 (sem correlação), até -1 (correlação negativa/inversa perfeita), sendo considerado que, em valor absoluto, até 0,33 indica relações fracas, entre 0,34 e 0,66 indica relações moderadas e acima de 0,67 indica relações fortes (Choudhury, 2009). Nos testes de validade de construto, foram utilizados testes paramétricos de diferenças de médias e o teste de independência do $\chi^{2}$.

\section{Resultados}

\section{Caracterizaçáo da amostra}

No total efetuaram-se 600 avaliações da MFS, dado que cada registo inclui três observações de avaliação do mesmo doente por três enfermeiros diferentes, no mesmo turno. Através da Tabela 2, pode verificar-se que as pessoas estavam na sua maioria internados em 
serviços de internamento médico-cirúrgico $(57,5 \%)$, predominando ligeiramente o sexo masculino (52\%), e tinham em média 72 anos com um desvio padrão (DP) de 13,7. A idade mais frequente foi 72 anos e 0 valor da mediana também de 72 anos com uma idade mínima de 23 anos e máxima de 98 anos. A grande maioria das pessoas (77,5\%) tinha idade superior a 65 anos. $O$ grupo etário com maior número de pessoas (34,0\%) foi o grupo dos 75 aos 84 anos. O principal motivo de admissão foi o internamento para intervenção cirurgia $(19,5 \%)$ e as patologias mais prevalentes que motivaram os restantes internamentos foram doenças infecciosas (16,5\%), doenças cardíacas (16,5\%) e doenças neurológicas (9,5\%). Quanto ao momento da aplicação da escala, a larga maioria foi aplicada no momento da admissão do doente (58\%), mas também foram aplicadas em momentos de reavaliação (40\%) e após queda (2\%).Os principais motivos de aplicação da escala foram a política definida pelas organizações estudadas em que está definida a aplicação da MFS, a idade do doente, a existência de quedas anteriores e, por fim, a agitação psicomotora. Constatou-se que os doentes estavam medicados com classes de fármacos associadas ao risco de queda, sendo as classes de fármacos mais prescritas os anti-hipertensores (38,1\%), os psicófarmacos (25,5\%) e os analgésicos estupefacientes (11,9\%). Constatou-se também que 30\% (60) dos doentes estavam medicados com dois fármacos associados ao risco de queda, 27,5\% (55) com um fármaco e 13,5\% (27) com três fármacos. A média de fármacos por doente associado ao risco de queda foi de 1,6 com um $D P$ de 1,2.

Tabela 2

Características dos doentes e dos internamentos $(n=200)$

\begin{tabular}{|c|c|c|c|}
\hline Variáveis & Características & $\mathrm{N}^{\mathrm{o}}$ & $\%$ \\
\hline \multirow[t]{2}{*}{ Hospital } & A & 110 & 55,0 \\
\hline & $\mathrm{B}$ & 90 & 45,0 \\
\hline \multirow[t]{5}{*}{ Serviços } & Medicina / Cirurgia & 115 & 57,5 \\
\hline & Especialidades médicas & 18 & 9,0 \\
\hline & Especialidades cirúrgicas & 27 & 13,5 \\
\hline & Unidade de cuidados intensivos & 15 & 7,5 \\
\hline & Cuidados continuados / paliativos & 25 & 12,5 \\
\hline \multirow[t]{2}{*}{ Sexo } & Masculino & 104 & 52,0 \\
\hline & Feminino & 96 & 48,0 \\
\hline \multirow[t]{7}{*}{ Idade } & Menos de 35 anos & 6 & 3,0 \\
\hline & De 35 a 44 anos & 5 & 2,5 \\
\hline & De 45 a 54 anos & 8 & 4,0 \\
\hline & De 55 a 64 anos & 26 & 13,0 \\
\hline & De 65 a 74 anos & 57 & 28,5 \\
\hline & De 75 anos a 84 anos & 68 & 34,0 \\
\hline & Mais de 84 anos & 30 & 15,0 \\
\hline \multirow[t]{12}{*}{ Motivo de internamento } & Cirurgia & 39 & 19,5 \\
\hline & Doença infecciosa & 33 & 16,5 \\
\hline & Doença cardíaca & 33 & 16,5 \\
\hline & Doença neurológica & 19 & 9,5 \\
\hline & Doença respiratória & 15 & 7,5 \\
\hline & Doença do aparelho digestivo & 12 & 6,0 \\
\hline & Doença oncológica & 12 & 6,0 \\
\hline & Reabilitação & 9 & 4,5 \\
\hline & Outros & 9 & 4,5 \\
\hline & Doença cérebro vascular & 8 & 4,0 \\
\hline & Doença vascular & 8 & 4,0 \\
\hline & Doença hepática & 3 & 1,5 \\
\hline \multirow[t]{3}{*}{ Momento da aplicação } & Admissão & 116 & 58,0 \\
\hline & Reavaliação & 80 & 40,0 \\
\hline & Após queda & 4 & 2,0 \\
\hline
\end{tabular}




\begin{tabular}{llcc} 
Classe dos fármacos** & Analgésicos estupefacientes (2.12) & 37 & 11,9 \\
& Antiepilépticos e anticonvulsivantes (2.6) & 19 & 6,1 \\
& Psicofármacos (2.9) & 79 & 25,5 \\
& Anti-hipertensores (3.4) & 118 & 38,1 \\
& Digitálicos (3.1.1) & 15 & 4,8 \\
& Insulinas (8.4.1) & 29 & 9,4 \\
Número de fármacos & Antidiabéticos orais (8.4.2) & 13 & 4,2 \\
& Sem fármacos & 45 & 22,5 \\
& Com um fármaco & 55 & 27,5 \\
& Com dois fármacos & 60 & 30,0 \\
& Com três fármacos & 27 & 13,5 \\
& Com quatro fármacos & 10 & 5,0 \\
\hline
\end{tabular}

* Grupos terapêuticos da classificação do Prontuário Terapêutico do Infarmed - 8

De acordo com a pontuação de risco, constatouse através da pontuação obtida, que 278 (46,3\%) observações foram avaliadas com alto risco de queda, 224 (37,3\%) com baixo risco e 98 (16,3\%) não apresentavam risco, sendo a média da pontuação de risco das 600 avaliações de 50,2 pontos com um DP de 24,2 .

A média do tempo de preenchimento do registo da escala foi de 5 minutos e não se registaram quaisquer problemas com a aplicação da escala.
A $71(35,5 \%)$ das pessoas foram aplicadas, como referido atrás, outras escalas, sendo as mais utilizadas a Escala de Coma de Glasgow e o Índice de Barthel. A Tabela 3 apresenta a distribuição das pontuações referentes a estes dois indicadores. Como se pode observar no quadro referido, o índice médio de gravidade da nossa amostra, medido pela Escala de Coma de Glasgow é muito baixo e o grau de dependência das pessoas, medida pelo Índice de Barthel, pode ser considerado moderado.

\section{Tabela 3}

Valores obtidos na Escala de Coma de Glasgow e no Índice de Barthel

\begin{tabular}{|c|c|c|c|c|c|c|c|}
\hline Indicador & & N. ${ }^{\circ}$ & $\%$ & Min & Max & Média & $\mathrm{DP}$ \\
\hline \multirow[t]{4}{*}{ Escala de Coma de Glasgow } & Soma & 49 & & 7 & 15 & 13,57 & $\pm 1,97$ \\
\hline & Abertura ocular & 49 & & 2 & 4 & 3,96 & $\pm 0,29$ \\
\hline & Resposta verbal & 49 & & 1 & 5 & 3,94 & $\pm 1,36$ \\
\hline & Resposta motora & 49 & & 4 & 6 & 5,67 & $\pm 0,66$ \\
\hline Índice de gravidade da & $0($ sem gravidade $)$ & 38 & 77,6 & & & & \\
\hline Escala de Coma de & 1 & 10 & 20,4 & & & & \\
\hline Glasgow & 2 & 1 & 2,0 & & & & \\
\hline Índice de Barthel & Soma & 39 & & 0 & 100 & 39,10 & $\pm 33,79$ \\
\hline \multirow[t]{5}{*}{ Grau de dependência de Barthel } & Total & 15 & 38,5 & & & & \\
\hline & Grave & 5 & 12,8 & & & & \\
\hline & Moderada & 6 & 15,4 & & & & \\
\hline & Leve & 11 & 28,2 & & & & \\
\hline & Independente & 2 & 5,1 & & & & \\
\hline
\end{tabular}

\section{Fiabilidade/Reprodutividade}

Na análise da fiabilidade, de uma forma geral, a análise da distribuição das frequências por indicador revelou uma grande concordância entre os enfermeiros (Tabela 4). As maiores divergências verificaram-se no item postura no andar e na transferência, entre o avaliador 2 e 3 no indicador debilitado, com uma frequência de 58 (29\%) versus 47 (23,5\%) e entre o avaliador 1 e 2, com uma frequência de 49 (24,5\%) versus 58 (29\%). No mesmo item, no indicador normal/acamado/imóvel, verifica-se entre o avaliador 1 e 2 uma frequência de 94 (47\%) versus 85 (42,5\%). 
Distribuição por item de concordância entre enfermeiros avaliadores

\begin{tabular}{|c|c|c|c|c|c|c|}
\hline \multirow{2}{*}{ Itens } & \multicolumn{2}{|c|}{$\begin{array}{c}\text { Avaliação } \\
1\end{array}$} & \multicolumn{2}{|c|}{$\begin{array}{c}\text { Avaliação } \\
2\end{array}$} & \multicolumn{2}{|c|}{ Avaliação 3} \\
\hline & N. ${ }^{\circ}$ & $\%$ & $\mathrm{~N}^{\mathrm{o}}$ & $\%$ & N. ${ }^{\circ}$ & $\%$ \\
\hline \multicolumn{7}{|l|}{ História de quedas } \\
\hline Sem quedas & 166 & 83,0 & 168 & 84,0 & 168 & 84,0 \\
\hline Com quedas & 34 & 16,0 & 32 & 16,0 & 32 & 16,0 \\
\hline \multicolumn{7}{|l|}{ Diagnóstico secundário } \\
\hline Sem diagnóstico secundário & 36 & 18,0 & 29 & 14,5 & 32 & 16,0 \\
\hline Com diagnóstico secundário & 164 & 82,0 & 171 & 85,5 & 168 & 84,0 \\
\hline \multicolumn{7}{|l|}{ Ajuda para caminhar } \\
\hline $\begin{array}{l}\text { Nenhuma/ajuda de enfermeiro/acamado/cadeira de } \\
\text { rodas }\end{array}$ & 145 & 72.5 & 152 & 76,0 & 151 & 75.5 \\
\hline Muletas/canadianas/bengala/andarilho & 27 & 13,5 & 25 & 12,5 & 24 & 12.0 \\
\hline Apoia-se no mobiliário para andar & 28 & 14,0 & 23 & 11,5 & 25 & 12.5 \\
\hline \multicolumn{7}{|l|}{ Terapia intravenosa } \\
\hline Sem terapia intravenosa & 51 & 25,5 & 51 & 25,5 & 50 & 25,0 \\
\hline Com terapia intravenosa & 149 & 74,5 & 149 & 74,5 & 150 & 75,0 \\
\hline \multicolumn{7}{|l|}{ Postura no andar e na transferência } \\
\hline Normal/acamado/imóvel & 94 & 47,0 & 85 & 42,5 & 89 & 44,5 \\
\hline Debilitado & 49 & 24,5 & 58 & 29,0 & 47 & 23,5 \\
\hline Dependente de ajuda & 57 & 28,5 & 57 & 28,5 & 64 & 32,0 \\
\hline \multicolumn{7}{|l|}{ Estado mental } \\
\hline Consciente das suas capacidades & 140 & 70,0 & 143 & 71,5 & 140 & 70,0 \\
\hline Esquece-se das suas limitações & 60 & 30,0 & 57 & 28,5 & 60 & 30,0 \\
\hline
\end{tabular}

Na Tabela 5, analisa-se o nível de concordância entre os observadores que variou entre 0,615 no item diagnóstico secundário e 0,964 no item bistória de quedas. A análise da média do coeficiente $\mathrm{K}$ demonstrou que o item ajuda para caminhar apresenta o nível de concordância mais baixo $(0,619)$ e o item história de quedas apresenta o nível de concordância mais elevado $(0,927)$. Nesta análise, verificou-se que os itens história de quedas, terapia intravenosa e estado mental apresentaram uma concordância quase perfeita. Os itens diagnóstico secundário, ajuda para caminhar e postura no andar e na transferência apresentaram um nível significativo de concordância.

O coeficiente de correlação intraclasse entre as avaliações dos três enfermeiros de cada doente revela um valor de concordância de 0,838 , associado a um intervalo de confiança a 95\% de ]0,800; 0,870[, existindo uma elevada concordância, da qual se pode inferir boa reprodutibilidade (Kramer \& Feinstein, 1981; Terwee et al.,2007).

Tabela 5

Nivel de concordância entre as observações

\begin{tabular}{lccc}
\hline Itens & Kapa & Erro padrão & Kapa médio \\
\hline História de quedas & & & 0,927 \\
Obs. 1 vs. Obs. 2 & 0,927 & 0,036 & \\
Obs. 1 vs. Obs. 3 & 0,964 & 0,026 & 0,657 \\
Obs. 2 vs. Obs. 3 & 0,963 & 0,026 & \\
Diagnóstico secundário & & & \\
Obs. 1 vs. Obs. 2 & 0,615 & 0,076 & 0,619 \\
Obs. 1 vs. Obs. 3 & 0,646 & 0,073 & \\
Obs. 2 vs. Obs. 3 & 0,710 & 0,070 & \\
Ajuda para caminhar & & 0,058 & \\
Obs. 1 vs. Obs. 2 & 0,639 & 0,062 & \\
Obs. 1 vs. Obs. 3 & 0,582 & 0,061 & \\
Obs. 2 vs. Obs. 3 & 0,635 &
\end{tabular}




\section{Terapia intravenosa}

Obs. 1 vs. Obs. 2

Obs. 1 vs. Obs. 3

Obs. 2 vs. Obs. 3

Postura no andar e na transferência

Obs. 1 vs. Obs. 2

Obs. 1 vs. Obs. 3

Obs. 2 vs. Obs. 3

Estado mental

Obs. 1 vs. Obs. 2

Obs. 1 vs. Obs. 3

Obs. 2 vs. Obs. 3

$\begin{array}{ll}0,921 & 0,032 \\ 0,881 & 0,039 \\ 0,934 & 0,029 \\ & \\ 0,637 & 0,045 \\ 0,642 & 0,045 \\ 0,639 & 0,045 \\ & \\ 0,867 & 0,039 \\ 0,786 & 0,048 \\ 0,795 & 0,047\end{array}$

0,912

0,639

0,816

índice de Barthel. Seria, por exemplo, de esperar que a pergunta 6 da MFS ('estado mental') estivesse significativamente relacionada com o índice total da Escala de Coma de Glasgow e, eventualmente a alguma das suas dimensões (Tabela 6).

Tabela 6

Médias ( $\pm D P$ ) da Escala de Coma de Glasgow

\begin{tabular}{lcccc}
\hline Estado Mental $\left(\mathrm{MFS}_{6}\right)$ & Abertura ocular & Resposta verbal & Resposta motora & Glasgow Total \\
\hline Consciente das suas capacidades & $4,00 \pm 0,00$ & $4,33 \pm 1,17$ & $5,79 \pm 0,59$ & $14,12 \pm 1,60$ \\
Esquece-se das suas limitações & $3,92 \pm 0,40$ & $3,56 \pm 1,45$ & $5,56 \pm 0,71$ & $13,04 \pm 2,17$ \\
U de Mann-Whitney & 288 & 186 & 245 & 180 \\
Significância & 0,327 & 0,014 & 0,131 & 0,011 \\
\hline
\end{tabular}

Verifica-se assim uma diferença significativa da pontuação da Escala de Glasgow para diferentes níveis de estado mental indicados pela MFS. Isto é, os indivíduos conscientes das suas capacidades obtêm maiores valores na Escala de Glasgow.

Por outro lado, as perguntas 8 ('transferir-se'), 9 ('deambular') e 10 ('escadas') do índice total de Barthel deveriam estar correlacionadas com as perguntas 3 ('apoio para caminhar') e 5 ('postura no andar e na transferência') da MFS; deveriam também estar correlacionadas, por serem associadas ao conceito de mobilidade e às atividades da vida diária. De facto, os indivíduos conscientes das suas capacidades $\left(\mathrm{MFS}_{6}=0\right)$ obtiveram uma resposta verbal mais orientada e um índice total de Glasgow significativamente mais elevado.

Relacionando agora com o Índice de Barthel, o item 8 da transferência (independente ou não) apresentou-se relacionado com a pergunta 3 ('necessitar ou não de apoio para caminhar') da MFS $\left(\chi^{2}=9,1 ; g l=1 ; p<0,005\right)$. Obtiveram-se semelhantes resultados com o item 9 de independência em deambular $\left(\chi^{2}=9,8: g l=1 ; p<0,005\right)$ e com o item 10 em utilizar as escadas $\left(\chi^{2}=3,6 \cdot g l=1 ; p<0,05\right)$.
Já a postura no andar e na transferência medida pela pergunta 5 da MFS não se mostrou relacionada nem com a independência do doente ao transferir-se nem em deambular nem mesmo em utilizar as escadas.

\section{Validade de construto}

Para testar a validade de construto, relacionou-se as pontuações médias da MFS com variáveis como o sexo, a idade, a existência de quedas anteriores e de agitação psicomotora, esperando-se alterações significativas das pontuações conforme o grupo analisado. Analisou-se também a sua relação com as três classes de fármacos mais prescritos aos doentes. Segundo experiência anterior, seria de esperar que os homens tivessem maior risco de quedas (porque, de facto, têm uma frequência maior de quedas), o mesmo acontecendo com os doentes mais idosos. Quedas anteriores e uma maior agitação psicomotora deveriam também estar associadas a um maior risco de queda avaliado pela MFS. Indivíduos aparentemente mais sedados deveriam, por fim, estar mais associados com um risco maior de queda. Apesar do risco de queda apresentado pelos homens ser, em média, superior ao das mulheres $(50,82 \pm 24,33$ vs. 48,54 $\pm 23,69 ; p<0,001)$, esta 
diferença é ligeira mas não significativa. Já em relação à idade, os doentes menos idosos apresentam um risco médio de quedas francamente mais baixo do que os mais idosos (34,67 $\pm 22,97$ vs. 54,09 $\pm 22,53$; $p<0,001$ ), o mesmo acontecendo com os que não tiveram historial de quedas no mesmo internamento ou episodio de urgência ou nos últimos três meses $(45,81 \pm 22,69$ vs. 68,82 $\pm 21,04 ; p<0,001)$, e com os que não apresentaram agitação psicomotora ( $47,14 \pm$ $23,39$ vs. $66,29 \pm 21,37 ; p<0,001)$.

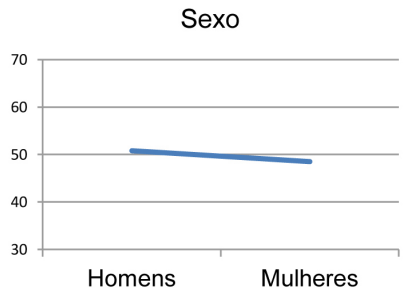

Historial de quedas

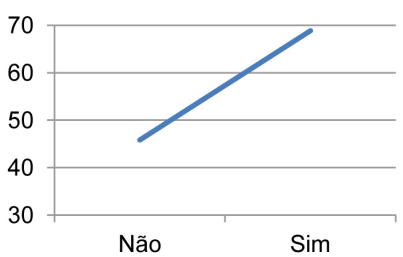

A figura 1 representa graficamente estas diferenças. Por fim, em relação às três classes de fármacos, mais prescritas ao doente de entre os sedativos (analgésicos estupefacientes, antiepilépticos e anticonvulsivantes, psicofármacos) e os sem poder sedativo (antihipertensores, digitálicos, insulinas e antidiabéticos orais), os doentes sedados apresentaram um maior risco de queda do que os não sedados (45,61 $\pm 23,50$ vs. $53,76 \pm 23,90 ; \mathrm{p}<0,05)$.

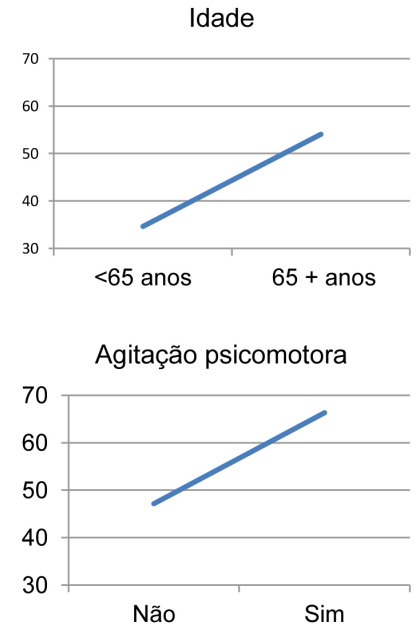

Figura 1. Relação entre o risco médio e as variáveis sexo, idade, história anterior de quedas e agitação psicomotora.

\section{Discussáo}

A utilização de escalas de avaliação do risco de queda, de rápido preenchimento, fiáveis, válidas, com nível significativo de concordância, desenvolvidas em contextos semelhantes ao estudado, que permitam identificar doentes com risco e estabelecer intervenções, são importantes para qualquer organização de saúde (Healy \& Scobie, 2007; Morse, 2009; Oliver et al., 2004; Perell et al., 2001), como parte integrante da sua política de garantia da qualidade dos cuidados prestados. A escala não-validada utilizada nos serviços onde decorreu o estudo, aproxima-se significativamente da versão agora validada.

Os internamentos onde a escala foi mais aplicada foram: internamentos médico-cirúrgicos, serviços também mais prevalentes em termos de aplicação da escala no estudo original de Morse (2009). Dos doentes que integraram este estudo, 77,5\% tinham uma idade superior a 65 anos, idade a partir da qual ocorrem cerca de 75\% das quedas, (Morse, 2009), tendo estas pessoas mais risco (Almeida, Abreu, \& Mendes, 2010) o que está relacionado com o facto de $46,3 \%$ dos doentes terem sido pontuados com alto risco de queda. O motivo de internamento pelo qual maior número de pessoas foi internado foi a intervenção cirúrgica. Estas pessoas têm mais risco de queda no seu pós-operatório devido a ficarem em algumas situações com um andar dependente de ajuda (Morse, 2009) e a tomarem fármacos para o controlo da dor, com efeito sedativo, tornando a pessoa mais vulnerável a uma queda.

As maiores divergências na avaliação dos doentes foram no item postura no andar e na transferência, sendo por isso importante que a escala seja sempre acompanhada de uma interpretação da pontuação em cada item por forma a uniformizar critérios de avaliação do doente (Morse, 2009). Neste item, as dúvidas surgem sobretudo relacionadas com as pessoas que 
utilizam cadeira de rodas para se movimentarem, sendo neste caso a pessoa avaliada em função da forma como efetua o processo de transferência entre a cadeira de rodas e a cama, podendo ter considerado para efeitos de pontuação como normal, se efetuar o processo sem dificuldades (Morse, 2009).

O tempo médio de utilização da escala para avaliação do risco esteve em conformidade com o que está descrito pela autora (Morse et al., 1989; Morse, 2009), considerando os enfermeiros que participaram no estudo que é de fácil preenchimento.

Neste artigo, apresentou-se o processo seguido para adaptar culturalmente para a língua portuguesa a MFS para avaliação do risco de quedas. Mostrou-se também evidência da sua fiabilidade, da sua validade e da sua concordância entre avaliadores, e da sua relação com outros instrumentos de medida. Apesar de ser uma escala há muito tempo frequentemente utilizada nas organizações de prestação de cuidados em Portugal, (Soares \& Almeida, 2008) pelo que sabemos, esta foi a primeira vez que foi validada em português.

A fiabilidade foi testada através do grau de concordância das pontuações fornecidas por três enfermeiros, independentemente uns dos outros. Este grau de concordância variou de 0,615 a 0,964 e o correspondente coeficiente de correlação interclasse foi de 0,838 , pelo que podemos afirmar que existe uma boa concordância entre os avaliadores na utilização da escala (Kramer \& Feinstein, 1981; Terwee et al., 2007). A revisão clínica foi realizada por um perito em Enfermagem e a validade facial garantida por um painel de peritos. A validade de critério foi demonstrada por correlações importantes entre as respostas às perguntas relevantes da MFS em comparação com as da Escala de Coma de Gasglow e o Índice de Barthel. A pergunta referente ao estado mental de consciência da MFS foi sensível à dimensão resposta verbal e ao índice total de Glasgow. Por outro lado, as perguntas do MFS relacionadas com o conceito de mobilidade e com as atividades da vida diária apresentaram-se relacionadas com os itens semelhantes do Índice de Barthel.

A validade de construto foi também confirmada com a evidência de maior risco de queda associado a idades mais avançadas, ao historial de quedas (Oliver et al., 2004), à agitação psicomotora (Perell et al., 2001) e à prescrição de fármacos com efeitos sedativos (Leipzig et al.,1999a; Leipzig et al.,1999b). O sexo masculino, com evidência de uma maior frequência de quedas, em outros estudos (Almeida et al., 2010) não apresentou, no presente estudo, qualquer influência na avaliação do risco de queda, o que nos faz levantar a hipótese de, com todas as restantes variáveis controladas, estes dois indicadores significarem conceitos distintos. Questões éticas fazem, no entanto, com que alguns tipos de validação não possam ser efetuados. Por exemplo, há presentemente uma tendência, perfeitamente justificada, para eliminar ao máximo o risco de quedas dos doentes nas organizações de saúde (Oliver et al., 2007). Isto faz com que os níveis de risco a partir dos quais há que iniciar ações tendentes a prevenir quedas sejam definidos e usados de uma forma cada vez mais conservadora (Morse, 2009) impedindo, de certo modo, a determinação da sensibilidade e da especificidade das escalas utilizadas. Fica, assim, beneficiado o doente, pois o pior que, em geral, pode acontecer, é a prestação de cuidados levar a cabo iniciativas de prevenção eventualmente desnecessárias.

\section{Conclusão}

Em conclusão, com base na metodologia seguida e de acordo com os nossos resultados, a versão portuguesa da MFS é semanticamente equivalente à versão original, conduzindo a bons níveis de fiabilidade e a níveis aceitáveis de validade. A sua utilização é assim recomendada na prestação de cuidados hospitalares em Portugal, podendo ser obtida através da consulta do Repositório de Instrumentos de Medição e Avaliação em Saúde (RIMAS) no endereço http:// www.uc.pt/org/ceisuc/RIMAS/Lista/Instrumentos/ MFS.

\section{Referências bibliográficas}

Almeida, R. A., Abreu, C. C., \& Mendes, A. M. (2010). Quedas em doentes hospitalizados: Contributos para uma prática baseada na prevenção. Revista de Enfermagem Referência, 3(2),163-172. doi:10.12707/RIII1016

Choudhury, A. (2009). Spearman rank correlation coefficient. Recuperado de http://www.experiment-resources.com/ spearman-rank-correlation-coefficient.html

Healey, F., \& Scobie, S. (2007). Slips, trips and falls in hospital: The third report from the Patient Safety Observatory. London, England: National Patient Safety Agency. 
Kohn, L. T., Corrigan, J. M., \& Donaldson, M. S. (2000). To err is human: Building a safer bealth system. Washington, WA: National Academy Press.

Kramer, M. S., \& Feinstein, A. R. (1981). The biostatistics concordance. Clinical Pharmacology and Therapeutics, 29(1),111-123.

Leipzig, R. M., Cumming, R. G., \& Tinetti, M. E. (1999a). Drugs and falls in older people: A systematic review and meta-analysis: I. Psychotropic drugs. Journal of the American Geriatrics Society, 47(1), 30-39.

Leipzig, R. M., Cumming, R. G., \& Tinetti, M. E. (1999b). Drugs and falls in older people: A systematic review and meta-analysis: II. Cardiac and analgesic drugs. Journal of the American Geriatrics Society, 47(1), 40-50.

Mokkink, L. B., Terwee, C. B., Patrick, D. L., Alonso, J., Stratford, P. W., Knol, D. L., ... de Vet, H. C. (2010). The COSMIN study reached international consensus on taxonomy, terminology, and definitions of measurement properties for health-related patient-reported outcomes. Journal of Clinical Epidemiology, 63(7), 737-745. doi: 10.1016/j. jclinepi.2010.02.006

Morse, J. M. (2006). The safety of safety research: The case of patient fall research. Canadian Journal of Nursing Research, 38(2),74-88.

Morse, J. M. (2009). Preventing patients falls: Establishing a fall intervention program (2nd ed.). New York, NY: Springer.

Morse, J. M., Morse, R. M., \& Tylko, S. J. (1989). Development of a Scale to Identify the Fall-Prone Patient. Canadian Journal on Aging, 8(4), 366-377.

Morse, J. M., Tylko, S. J., \& Dixon, H. A. (1987). Characteristics of the fall-prone patient. The Gerontologist, 27(4), 516-522.

Oliver, D. (2008). Falls risk-prediction tools for hospital inpatients. Time to put them to bed? Age and Ageing, 37(3), 248-250. doi:10.1093/ageing/afn088
Oliver, D., Britton, M., Seed, P., Martin, F. C., \& Hopper, A. H. (1997). Development and evaluation of evidence based risk assement tool (STRATIFY) to predict which elderly inpatients will fall: Case-control and cohort studies. British Medical Journal, 315(7115), 1049-1053.

Oliver, D., Connelly, J. B., Victor, C. R., Shaw, F. E., Whitehead, A., Genc, Y., ... Gosney, M. A. (2007). Strategies to prevent falls and fractures in hospitals and care homes and effect of cognitive impairment: Systematic review and meta-analyses. British Medical Journal, 334(7584), 82-85. doi:10.1136/ bmj.39049.706493.55

Oliver, D., Daly, F., Martin, F., \& McMurdo, M. (2004). Risk factors and risk assessment tools for falls in hospital in-patients: A systematic review. Age and Ageing, 33(2), 122-130. doi:10.1093/ageing/afh017

Perell, K. L., Nelson, A., Goldman, R. L., Prieto-Lewis, N., \& Rubenstein L. Z. (2001). Fall risk assessment measures: An analytic review. Journal of Gerontology, 56(12), 761-766. doi:10.1093/gerona/56.12.M761

Soares, M. E., \& Almeida, M. R. (2008). Acidentes com macas e camas em estabelecimentos bospitalares, envolvendo a queda de doentes (Relatório No 319/08). Lisboa: Inspecção Geral das Actividades em Saúde.

Terwee, C. B., Bot, S. D., de Boer, M. R., van der Windt, D. A., Knol, D. L., Dekker, J., ... de Vet, H.C. (2007). Quality criteria were proposed for measurement properties of health status questionnaires. Journal of Clinical Epidemiology, 60(1), 3442.

Wild, D., Grove, A., Martin, M., Eremenco, S., McElroy, S., VerjeeLorenz, A., \& Erikson, P. (2005). Principles of good practice for the translation and cultural adaptation process for patient-reported outcomes (PRO) measures: Report of the ISPOR Task Force for Translation and Cultural Adaptation. Value In Health, 8(2), 94-104. 
